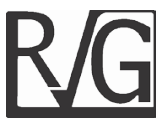

\title{
Orientación al Cliente, Tecnologías de Información y Desempeño Organizacional: Caso empresa de consumo masivo en Chile ${ }^{*}$
}

\author{
Valenzuela Fernández, Leslier Maureen ${ }^{* \star}$ \\ Martínez Troncoso, Carolina Andrea ${ }^{* \star}$
}

\section{Resumen}

El objetivo de la presente investigación es determinar si efectivamente una organización que ha alcanzado una posición de líder en un determinado mercado adopta tecnologías de información ( $\mathrm{TI})$ y una gestión orientada al cliente $(\mathrm{OC})$ con el fin de generar inteligencia de negocio para lograr un mejor desempeño organizacional. Asimismo, se contribuye con una propuesta de un modelo que explica las relaciones entre constructos fiables de Orientación al Cliente, Tecnologías de Información y Desempeño Organizacional. Se aporta evidencia empírica de una empresa líder del sector de consumo masivo en Chile, desde la perspectiva de la oferta y la demanda. El estudio se aborda en dos fases, la primera mide la relación entre las tres variables descritas, y en la segunda la satisfacción del cliente sobre dos marcas de la empresa líder. Los resultados del estudio apoyan relaciones positivas entre Orientación al Cliente, Tecnologías de Información y Desempeño Organizacional. Sin embargo, los consumidores podrían no estar percibiendo mejoras en el producto y servicio recibido (percepción de satisfacción de consumo) a pesar del enfoque de gestión por parte de la organización en función del valor del cliente.

Palabras clave: Tecnologías de Información; orientación al cliente; desempeño organizacional; percepción de consumo.

Recibido: 25.11.13. Aceptado: 29.04.15

* Investigación derivada de la Etapa 1 Proyecto Fondecyt N 11100163. Investigador Responsable: Leslier Valenzuela Fernández, Ph.D.

** Doctora en Marketing, Universidad Complutense de Madrid, MBA University of Guelph, Canadá. Profesor Asistente de Marketing, Departamento de Administración, Facultad de Economía y Negocios, Universidad de Chile, Ivalenzuela@unegocios.cl

*** Magíster en Marketing, Universidad de Chile. Profesora Asistente, Facultad de Economía y Negocios, Universidad Finis Terrae, cmartinezt@uft.cl 


\title{
Client orientation, information technologies and organizational performance: Mass consumption company case in Chile
}

\begin{abstract}
The aim of the present research is to determine if effectively an organization that has reached a leading position in a particular market embraces information technologies (IT) and a client oriented (CO) management with the propose of generating business intelligence to achieve a better organizational performance. Likewise, it contributes with a model proposal that explains the association between reliable constructs of Client Orientation, Information Technologies and Organizational Performance. Empiric evidence of a leading company from the mass consumption sector in Chile through the supply and demand perspective is provided. The study is approached in two phases; the first, measures the association between the three variables described and the second, the clients satisfaction about two brands of the lead company. The study results support positive associations between Client Orientation, Information Technologies and Organizational Performance. However, the consumers may not be perceiving improvements on the product and service received (perception of consumption satisfaction) despite the organizations management approach depending on the clients value.
\end{abstract}

Key Words: Information technologies; cilents orientation; organizational performance; consumption perception

\section{Introducción}

Bajo el entorno altamente competitivo en que deben sobrevivir las empresas en la actualidad, la orientación al cliente es un factor clave para que los negocios triunfen (Jaramillo y Grisaffe, 2009; Valenzuela, 2010; Valenzuela et al, 2010). En este contexto, las grandes compañías de consumo masivo para estimular la demanda, invierten cientos de miles de millones de dólares en desarrollar estrategias de marketing orientadas al cliente que permitan cocrear valor y establecer relaciones de largo plazo (Coley et al, 2010; Hunter y Perreault 2007; Prahalad y Ramaswamy, 2004), situando al cliente en el centro de la estrategia de negocio, pasando de "actuar para el cliente" a "actuar con el cliente" (Valenzuela, 2007).

En la última década, varios investigadores han estudiado la relación entre la orientación al cliente y los resultados organizacionales, encontrando en su mayoría una relación positiva (Homburg et al, 2011; Jaramillo y Grisaffe, 2009; Franke y Park 2006; Macintosh, 2007; Kirca et al, 2005). En efecto, una filosofía de gestión con visión de equipo de co-generación de valor entre todas las partes involucradas que facilite la asociatividad o colaboración con los stakeholders se tornaría fundamental en la obtención de mejores resultados organizacionales (Valenzuela, 2010).

Por otra parte, para lograr satisfacer las necesidades del cliente y gestionar una relación de largo plazo es necesario poseer competencias tecnológicas (Hortinha et al, 2011; Danneels 2002; Gatignon y Xuereb 1997; Yalcinkaya et al, 2007; Zhou et al, 2005), las cuales según Danneels (2002) bridan a las empresas habilidades para diseñar y producir un producto nuevo con atributos técnicos. 
Orientación al Cliente, Tecnologías de Información y Desempeño... Valenzuela Fernández, Leslier Maureen y Martínez Troncoso, Carolina Andrea

Las tecnologías de información (TI) por parte de las empresas constituiría un factor determinante de competitividad impactando en los resultados organizacionales (Meléndez y Moreno, 2012; Yang y $\mathrm{Fu}, 2007$ ), puesto que la adopción de TI sería un facilitador del establecimiento de relaciones de cooperación con diferentes agentes del entorno (Deeds y Rothaermel, 2003; Yadav y Varadarajan, 2005). Una buena gestión en la adopción de competencias tecnológicas permitiría la generación de conocimiento e inteligencia de negocio, lo cual contribuye en la toma de decisiones estratégicas para generar mayor valor para el cliente y que por tanto, potencien el desarrollo de ventajas competitivas (Brady et al, 2002, Valenzuela y Martínez, 2012).

Por consiguiente, la justificación e importancia de esta investigación, en términos de contribución a la teoría y práctica de marketing y ventas, yace en que este estudio sugiere que la gestión orientada en función del valor del cliente y la adopción de TI, especialmente en el sector servicio y en este caso de estudio, sector retail, juegan un rol fundamental en explicar el desempeño en la gestión de marketing y ventas y su impacto en el desempeño organizacional.

Bajo esta óptica, surgen las siguientes interrogantes: ¿las organizaciones que efectivamente han alcanzado un alto rendimiento estarían gestionando en función del cliente?; ¿estas organizaciones estarían adoptando las tecnologías de información con el fin de generar inteligencia de negocios? ¿Efectivamente existe una posible relación positiva entre la orientación al valor del cliente y la adopción de tecnologías de información con un mejor desempeño organizacional? Y de ser así ¿los clientes perciben las mejoras en la entrega del producto o servicio y por lo tanto, se encuentran más satisfechos con el proveedor?

Para dar respuesta a las interrogantes anteriores, el objetivo central de esta investigación es determinar si efectivamente una organización que ha alcanzado una posición de líder en un determinado mercado adopta tecnologías de información (TI) y una gestión orientada al cliente $(\mathrm{OC})$ con el fin de generar inteligencia de negocio para lograr un mejor desempeño organizacional. La investigación contribuye con la propuesta de un modelo que explica las relaciones entre constructos fiables de Orientación al Cliente (OC), Tecnologías de Información (TI) y Desempeño Organizacional (DO), a través del testeo de hipótesis aplicado a la oferta.

El estudio se aborda en dos fases, la primera mide la relación entre las tres variables descritas, y la segunda la satisfacción del cliente sobre dos marcas de la empresa líder evaluada en este estudio. Esto se realiza por medio de un cuestionario aplicado a altos directivos y empleados de una empresa chilena líder del sector de consumo masivo, así como a consumidores de dos marcas de la empresa evaluada para la segunda fase.

\section{Orientación al cliente (OC)}

En los últimos años, el concepto de OC se ha convertido en uno de los temas claves de estudio en la literatura en marketing y administración de empresas (Homburg et al, 2011). Esto se debe a cuatro razones principales.

Primero, la gestión de la cartera de clientes como un activo estratégico es una de las maneras más eficientes 
de administrar y asignar los recursos limitados de la empresa (Valenzuela y Castillo, 2007). Segundo, la OC influiría positivamente en la satisfacción y confianza del cliente, lo que a su vez, tendría una influencia positiva en la retención y generación de relaciones rentables de largo plazo con los clientes (ljaz y Mehmood, 2011; Kumar et al, 2006; Shah et al, 2006). Tercero, aumentaría la rentabilidad del negocio a través del crecimiento de las ventas en el tiempo (Jaramillo y Grisaffe, 2009). Cuarto, la OC generaría ventajas competitivas (Kumar et al, 2006; Valenzuela y Torres, 2008) que pueden contribuir de forma importante al éxito empresarial (Slater y Olson 2001; Atuahene-Gima et al, 2005).

Por tanto, conocer de mejor manera al cliente en función de su valor, provocaría que las empresas sean conscientes de las oportunidades del mercado, mejoren sus procesos y la asignación de recursos (Hortinha et al, 2011; Yalcinkaya et al, 2007) y por ende, su desempeño organizacional. Así se formula la primera hipótesis del estudio:

H1: El grado de orientación al cliente (OC) por parte de la empresa se relaciona positivamente con el desempeño organizacional (DO)

En este sentido, Zeithaml (1988) define el "valor percibido por el cliente" como la percepción respecto al intercambio de lo que se recibe de la compañía y lo que se da a cambio. Del mismo modo, Kotler (1999) señala que "es la diferencia entre el valor total que recibe el cliente y el costo total en el que incurre". Por su parte, Valenzuela (2007) define el valor recibido como "el valor que actualmente experimenta el cliente a través de interacciones específicas con el producto" y el valor deseado como "el total de bondades de los atributos del producto, y las consecuencias resultantes, tanto positivas como negativas, monetarias $y$ no monetarias, que el cliente quiere $y$ espera que ocurra". Se ha demostrado que muchas veces existen diferencias entre el valor percibido y recibido por los clientes y entre el valor recibido y deseado por ellos (Flint y Woodruf, 2001), por lo que es fundamental para las compañías comprender al cliente, de manera de poder satisfacer de mejor manera sus necesidades y generar mayor rentabilización en los segmentos objetivo (Valenzuela, 2010).

Entonces ¿qué características determinarían que una empresa se encuentre orientada al cliente? La adopción de una cultura organizacional orientada al cliente (COC) requiere de una comunicación expedita dentro de la organización, de manera que toda la información sobre la satisfacción y la rentabilidad de sus clientes se encuentre disponible para todas las áreas (Narver y Slater, 1990; Deshpande et al, 1993 y Valenzuela, 2007), es decir, que exista una coordinación interfuncional comprometida en la creación de condiciones necesarias que ofrezcan valor a los clientes y, en general, a todos los agentes de la cadena de valor (Narver y Slater, 1990). No obstante, Hortinha et al, (2011), Atuahene-Gima et al, (2005), Baker y Sinkula (2007) y Zhou at al, (2005) apoyan que la adopción de una filosofía empresarial orientada al cliente por sí sola no llevaría directamente a una mejora en el desempeño organizacional, a menos de que existan las competencias tecnológicas necesarias para que la organización desarrolle un cierto nivel de aprendizaje.

Lo anterior deriva en la siguiente hipótesis de estudio:

H1a: El grado de cultura organizacional orientada al cliente 
Orientación al Cliente, Tecnologías de Información y Desempeño... Valenzuela Fernández, Leslier Maureen y Martínez Troncoso, Carolina Andrea

(COC) se relacionaría positivamente con el grado de orientación al valor del cliente $(\mathrm{OC})$ que exista en la gestión empresarial.

Por otra parte, la orientación al cliente supone una mayor comprensión por parte de la organización sobre el mercado objetivo, acorde a la creación de un valor superior para los mismos (Narver y Slater, 1990). Para poder diseñar y desarrollar todas las acciones destinadas a conseguir los objetivos empresariales, la empresa necesita disponer de un profundo conocimiento del entorno y de todos los agentes que lo conforman (Frishammar, 2005).

Según Elg (2002), el proceso de generación, diseminación y uso de una inteligencia de negocio favorece la cohesión entre las empresas que conforman una red, mejorando su capacidad de adaptación y su capacidad de respuesta a los retos del entorno. Por tanto, el conocimiento del cliente (CC) de una manera sistemática y confiable sería la base de un buen sistema de gestión en función del cliente (Hortinha et al, 2011). El conocimiento de las preferencias, necesidades y la orientación constante al aprendizaje son las características fundamentales de organizaciones orientadas al cliente (Berger et al, 2002). Así se deriva la siguiente hipótesis

H1b: El grado de comprensión y conocimiento sobre los clientes y/o consumidores (CC) se relacionaría positivamente con el grado de orientación al valor del cliente (OC) que exista en la gestión empresarial.

Por otra parte, cabe destacar además la existencia de investigaciones empíricas respecto de la OC desde la perspectiva de sus empleados, en éstas se plantea que las organizaciones deberían estimular activamente a sus empleados a adoptar comportamientos en busca de beneficiar al cliente (Zablah, et al, 2012; Homburg et al, 2011; Liang et al, 2010; Baxter y Matear, 2004; Brown et al, 2002). Y que dicho desempeño puede ser visto como resultado de las capacidades, personalidad, actitudes y habilidades de un empleado para retener a sus clientes (Churchill et al, 1985; Plank y Reid, 1994). Un alto grado de orientación al cliente por parte de los empleados (GOCE), ayudaría a crear relaciones estables, aumentar la cooperación entre cliente-empresa (Hunter y Perreault 2007) y crear valor para al cliente (Brady y Cronin, 2001). Asimismo, la orientación al cliente por parte de los vendedores conduciría a mayores volúmenes de venta, por medio de incrementos en venta cruzada, retención de clientes y compras inmediatas (Jones et al, 2003).

Lo anterior deriva en las siguientes hipótesis de estudio:

H1c: El grado de orientación al cliente que tengan los empleados (GOCE) se relacionaría positivamente con el grado de orientación al valor del cliente (OC) que exista en la gestión empresarial.

\section{Tecnologías de información (TI)}

Según investigación realizada por Hortinha et al (2011) el tradeoff existente entre la OC y las tecnologías de Información, también llamada "orientación a la tecnología" o "competencias tecnológicas", señala que no hay consenso cuál de estas variables tendría un mayor impacto en el desempeño organizacional.

La adopción de herramientas tecnológicas por parte de las empresas, ha permitido facilitar el uso de bases de 
datos e información de los clientes con el fin de generar conocimiento (Lam et al, 2001). La automatización de los procesos ayudaría a la generación de inteligencia de negocios permitiendo tratar a los clientes adecuada y oportunamente para la toma de decisiones (Jiménez y Martínez, 2006).

Acorde a García et al, (2007) las empresas pueden adoptar estratégicamente las $\mathrm{TI}$ con tres principales fines: 1) mejorar la eficiencia interna de la empresa; 2) mejorar la atención a los clientes actuales; y 3) definir nuevos mercados y oportunidades de negocio. Una de las aplicaciones más importantes es la gestión de relaciones con clientes (Customer Relationship Management, CRM), es decir, seleccionar y gestionar la relación con los mejores clientes para optimizar su valor en el largo plazo, mediante la aplicación de softwares para procesar la información y así desarrollar una relación permanente en el tiempo (Meléndez y Moreno, 2012; Valenzuela, 2007). Bajo esta perspectiva, la adopción de CRM permitiría identificar y conocer mejor a los clientes (su rentabilidad actual y futura, su grado de satisfacción, fidelización, entre otros aspectos) y personalizar con mayor precisión los productos o servicios (Reinartz et al, 2004).

El presente estudio se basa en el Modelo de Aceptación de Tecnología (TAM) de Davis (1989). Este modelo sugiere que cuando a los empleados se les presenta una nueva tecnología, una serie de factores influyen en su decisión respecto de cómo y cuándo las van a utilizar (Orantes, 2011). Estos factores son: 1) utilidad Percibida definida como "el grado en que una persona cree que utilizando un determinado sistema mejorará su rendimiento en el trabajo" (Davis, 1989). Los individuos evaluarían las consecuencias de sus comportamientos (el uso de $\mathrm{TI}$ ) teniendo en cuenta las recompensas potenciales (Porter y Lawler, 1968). Y 2) facilidad de uso Percibida la cual se define como el "grado en que una persona cree que utilizando un sistema en particular, realizará menos esfuerzo al desempeñar una tarea" (Davis, 1989). Las premisas originales del Modelo TAM plantean que existe una influencia por parte de la Utilidad Percibida y la Facilidad de Uso Percibida hacia la Actitud de Uso de $\mathrm{TI}$, la cual es entendida como "la disposición que tenga el usuario ante una determinada tecnología". Existiría por tanto una relación directa entre la actitud hacia el uso de tecnologías y la intención de usarlas (Orantes, 2011).

Para que la TI por parte de la empresa tenga éxito, y por tanto, se genere inteligencia de negocio gracias a su uso es importante tener en cuenta algunas variables externas. Según Fullam (1999) tener una cultura organizacional orientada a las $\mathrm{TI}$ podría crear un clima a favor o en contra del uso de $\mathrm{TI}$. Riascos et al, (2009) menciona además que otro de los factores principales para el éxito de la implementación de las TI es el perfil de los empleados en relación a la adopción de TI (PEATI). Existirían dos tipos de perfiles: aquellos empleados que se resisten a involucrarse en nuevos métodos, apelando a que las herramientas que han utilizado durante tanto tiempo han funcionado bien y no consideran necesario hacer cambios y los trabajadores con mentalidad más abierta, que están dispuestos a integrarse en busca de mejorar las técnicas existentes. Estos últimos perciben mayor facilidad en el uso de las herramientas y comprenden realmente 
Orientación al Cliente, Tecnologías de Información y Desempeño... Valenzuela Fernández, Leslier Maureen y Martínez Troncoso, Carolina Andrea

los beneficios que las tecnologías proveen a la organización y a su gestión, y por ende, tienen una mayor disposición hacia el uso de estos sistemas.

Finalmente, desde los años noventa se ha tratado de indagar en el impacto de las $\mathrm{TI}$ en el desempeño organizacional, la evidencia existente hasta el momento, no ha sido concluyente, aunque principalmente se ha encontrado evidencia de una relación positiva entre adopción entre TI y DO (Brynjolfsson y Hitt, 1996, Kohli y Devaraj, 2003, Mukhopadhyay et al, 1995). No obstante, la mayoría de los estudios han sido aplicados en países anglosajones y desarrollados.

Derivado de estos planteamientos se han establecido las siguientes Hipótesis de estudio:

H2: El grado de adopción de tecnologías de información (TI) se relacionaría positivamente con el grado de orientación al valor del cliente (OC) que tenga la empresa.

H2a: El grado de cultura orientada al uso de TI (COATI) que tenga la empresa se relacionaría positivamente con la facilidad percibida en el uso de TI (FUATI) que presenten los miembros de una empresa.

H2b: El grado de cultura orientada al uso de TI (COATI) que tenga la empresa se relacionaría positivamente con el grado de utilidad percibida en el uso de TI (UPATI).

H2c: El perfil de los empleados en relación al uso de $\mathrm{TI}$ (PEATI) se relaciona positivamente con la facilidad percibida en el uso de TI (FUATI) que presenten los miembros de una empresa.

H2d: El perfil de los empleados en relación al uso de TI (PEATI) se relaciona positivamente con el grado de utilidad percibida en el uso de TI (UPATI) que presenten los miembros de una empresa.

H2e: El grado de utilidad percibida en el uso de $\mathrm{TI}$ (UPATI) se relaciona positivamente con la actitud hacia el uso de $\mathrm{TI}$ (AUATI) que presenten los miembros de la empresa.

H2f: La facilidad percibida en el uso de $\mathrm{TI}$ (FUATI) se relaciona positivamente con la actitud hacia el uso de TI (AUATI) que presenten los miembros de una empresa.

H2g: La actitud que se tenga hacia el uso de $\mathrm{TI}$ (AUATI) se relacionaría positivamente con el grado de adopción de $\mathrm{TI}(\mathrm{TI})$ que tenga una empresa.

H3: El grado de adopción de tecnologías de información ( $\mathrm{TI}$ ) se relacionaría positivamente con el desempeño organizacional (DO)

\section{Desempeño organizacional (DO)}

Una de las principales discusiones expuestas en la literatura se refiere a cómo medir el constructo de "desempeño organizacional" (Araya et al, 2007). Tradicionalmente se han utilizado medidas financieras y/o objetivas, como por ejemplo: la rentabilidad sobre la inversión (ROI), la rentabilidad sobre el patrimonio (ROE), niveles de venta, frecuencia de compra, el comportamiento de compra y valor de mercado (Reinartz y Kumar, 2003). Sin embargo, en los últimos años se han agregado variables no financieras $y / o$ subjetivas, básicamente porque permiten captar información del ambiente de negocios que las medidas financieras no pueden (Hoque, 2004). Las medidas no financieras, también permiten analizar dimensiones de la posición competitiva, como la percepción que tienen los 
clientes o empleados y variaciones de la cuota de mercado (Kaplan y Norton, 1996). En la presente investigación se utilizó como medida de DO "las percepciones de los empleados respecto del desempeño de la organización".

Dado lo anterior, se deriva el siguiente modelo explicativo: (Diagrama 1).

\section{Diagrama 1}

\section{Modelo Explicativo}

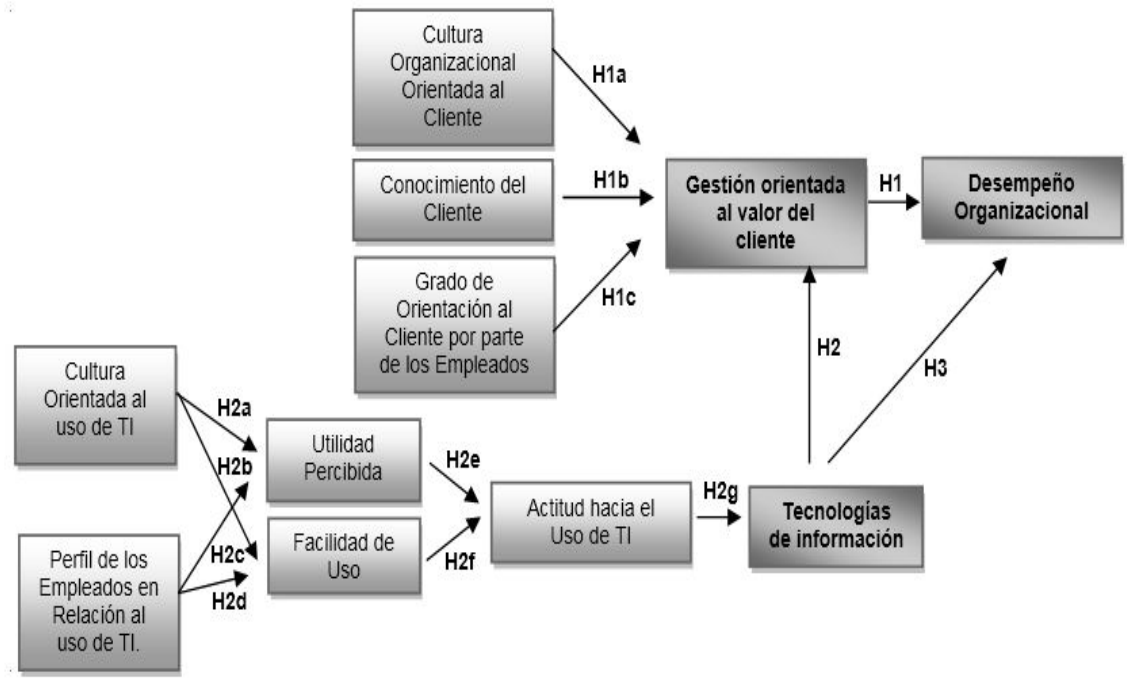

Fuente: Elaboración Propia (2012)

\section{Percepción de satisfacción de consumo}

Según Flint y Woodruf (2001) es muy común que existan diferencias entre el valor deseado y percibido por los clientes y el que efectivamente reciben. Muchas veces los empleados podrían percibir que su trabajo es óptimo, y que por tanto los clientes una vez recibida la prestación se retiran satisfechos con el producto o servicio. ¿Pero eso realmente ocurre? La Satisfacción de los Clientes (SC), se comprende como un estado profundamente emocional de una experiencia de compra o consumo en relación de la expectativa del cliente (Oliver, 1997). Toda empresa que logre que un cliente esté satisfecho obtendrá probablemente una difusión bocaoído positiva hacia otras personas (valor de referencia) y reiteración en la compra. Por consiguiente, empresas con mayor grado de satisfacción de 
Orientación al Cliente, Tecnologías de Información y Desempeño... Valenzuela Fernández, Leslier Maureen y Martínez Troncoso, Carolina Andrea

sus clientes podrían esperar mayores beneficios (Anderson et al, 1994), pero no necesariamente es así, ya que la lealtad de los consumidores es cada vez más difícil debido a la proliferación de productos, acceso a la información, la alta competencia que hace que los productos tiendan a la indiferenciación, entre otros factores. Por lo tanto el valor de la experiencia con las marcas se tornaría cada vez más relevante para aumentar la lealtad de los consumidores.

\section{Influencia de Orientación al Cliente, Tecnologías de Información en el Desempeño Organizacional}

En esta sección se presentan los resultados de una empresa líder del sector de consumo masivo en Chile obtenidos en las dos fases del estudio, siendo la primera fase, el estudio de las relaciones entre la Orientación al Cliente (OC), Tecnologías de Información (TI) y Desempeño Organizacional (DO). Mientras que la segunda es la verificación de la Satisfacción del cliente.

La primera fase contempla un análisis cuantitativo transversal simple con una estrategia de obtención de datos vía presencial. Se diseña un instrumento (cuestionario autoadministrado) con un total de 50 indicadores de escalas ya validadas en disertaciones teóricas previamente mencionadas. Estos indicadores se midieron a través de una escala Likert de 7 puntos, 3 constructos principales: Orientación al Cliente (OC); Tecnologías de Información (TI) y Desempeño Organizacional (DO). Previamente se realizó un pre-test del instrumento con el fin de depurarlo. Los criterios utilizados para examinar la unidimensionalidad, validez convergente y fiabilidad de los constructos fueron: Kayser-Meyer-Olkin mayor que 0,6, Eigenvalue mayor que 1, el Test de Esfericidad de Bartlett menor que 0,5 , cargas factoriales mayores a 0,7, y Alpha de Cronbach mayor que 0,7.

El muestreo fue del tipo no probabilístico por juicio. Se trabajó con una empresa líder del consumo masivo, una multinacional que cuenta con un portafolio de 25 marcas a nivel mundial. La elección del sector radica en la importancia económica que tiene la industria del consumo masivo en Chile. El año 2012 este sector industrial vendió US\$ 52.990 millones, las cuales se estiman para el 2015 aumenten a los US\$ 60.690 millones, es decir, las ventas del sector se elevarían en un $14,5 \%$ entre 2011 y $2015^{1}$.

En la segunda fase, con el fin de comprobar que la calidad de servicio entregada por los empleados a los clientes es efectivamente percibida (es decir, el cliente se encuentra satisfecho con el producto o servicio adquirido), se realizó un análisis cuantitativo transversal simple con una estrategia de obtención de datos vía online.

El instrumento aplicado fue un cuestionario auto-administrado con un total de 15 indicadores, los cuales se midieron a través de una escala Likert de 7 puntos, para medir el constructo: Satisfacción del Cliente (SC). El procedimiento de muestreo fue no probabilístico por conveniencia. Los encuestados fueron 105 consumidores para la marca 1 (uso femenino) y 102 para la marca 2 (uso masculino) debido

1 Datos otorgados por la consultora londinense Business Monitor International (2012). 
a que la empresa de consumo masivo en estudio vende productos dirigidos a distintos segmentos.

\subsection{Orientación al Cliente (OC); Tecnologías de Información (TI) y Desempeño Organizacional (DO)}

En esta primera fase del estudio, la muestra consistió en un total de 49 altos directivos y personal de marketing y ventas de una importante empresa del sector consumo masivo. La distribución por género correspondió a un 46,9\% de hombres y un $53,1 \%$ mujeres. Mayoritariamente menores de 34 años $(60 \%)$, con un nivel dominante de estudios universitarios completos $(45 \%)$ de los cuales un $10,2 \%$ poseía estudios a nivel de postgrado. La caracterización del área de desempeño de los encuestados fue: empleados pertenecientes a las áreas de Ventas (53,2\%), Marketing $(22,4 \%)$, Gerencia de Ventas (6,1\%), Gerencia de Operaciones $(2 \%)$ y Personal en el punto de venta (16,3\%). Respecto al tiempo de permanencia en la empresa, más del $70 \%$ de los encuestados no supera los 5 años en el cargo.

Con respecto a los análisis de fiabilidad y factorial exploratorio, los Alfas de Cronbach para cada uno de los constructos analizados superó los 0,7 con excepción del constructo GOCE el cual arrojó un valor de 0,629 (Tabla 1) mostrando consistencia interna. $\mathrm{El}$ análisis factorial exploratorio arrojó once factores que explicaron más del $70 \%$ de la varianza. Al realizar un análisis individual por constructo se observó que todos superaron el nivel mínimo de Alpha de Cronbach exigido y se agruparon en un sólo factor (unidimencionalidad).

Tabla 1

Detalle de Alphas de Cronbach por Constructo

\begin{tabular}{ccc}
\hline Constructo & Alpha de Cronbach & Media Constructo \\
\hline GOCE & 0,629 & 5,985 \\
COC & 0,795 & 5,878 \\
CC & 0,857 & 5,941 \\
COATI & 0,938 & 6,375 \\
PEATI & 0,872 & 6,267 \\
UPATI & 0,875 & 6,139 \\
FUATU & 0,945 & 5,828 \\
UATI & 0,940 & 6,293 \\
OC & 0,729 & 5,959 \\
TI & 0,738 & 5,396 \\
DO & 0,864 & 5,686 \\
\hline
\end{tabular}

Fuente: Elaboración Propia (2012) 
Orientación al Cliente, Tecnologías de Información y Desempeño...

Valenzuela Fernández, Leslier Maureen y Martínez Troncoso, Carolina Andrea

Para validar las hipótesis, se analizaron las correlaciones con el fin de (positiva o negativa) entre los diferentes observar la posible influencia o relación constructos (Tabla 2).

Tabla 2

Matriz de Correlaciones por Constructo

\begin{tabular}{|c|c|c|c|c|c|c|c|c|c|c|c|}
\hline & GOCE & $\mathrm{COC}$ & CC & COATI & PEATI & UPATI & FUATI & UATI & $O C$ & TI & DO \\
\hline GOCE & 1,000 & & & & & & & & & & \\
\hline $\mathrm{COC}$ & 0,182 & 1,000 & & & & & & & & & \\
\hline CC & 0,187 & 0,755 & 1,000 & & & & & & & & \\
\hline COATI & 0,093 & 0,544 & 0,581 & 1,000 & & & & & & & \\
\hline PEATI & 0,227 & 0,335 & 0,302 & 0,562 & 1,000 & & & & & & \\
\hline UPATI & 0,179 & 0,539 & 0,420 & 0,637 & 0,815 & 1,000 & & & & & \\
\hline FUATI & 0,144 & 0,249 & 0,160 & 0,297 & 0,741 & 0,609 & 1,000 & & & & \\
\hline UATI & 0,203 & 0,349 & 0,238 & 0,518 & 0,768 & 0,788 & 0,496 & 1,000 & & & \\
\hline OC & 0,463 & 0,819 & 0,895 & 0,542 & 0,392 & 0,488 & 0,214 & 0,350 & 1,000 & & \\
\hline TI & 0,293 & 0,542 & 0,521 & 0,697 & 0,891 & 0,886 & 0,706 & 0,770 & 0,595 & 1,000 & \\
\hline DO & 0,388 & 0,546 & 0,474 & 0,380 & 0,370 & 0,484 & 0,298 & 0,239 & 0,583 & 0,501 & 1,000 \\
\hline
\end{tabular}

Fuente: Elaboración Propia (2012)

A partir de los resultados de la Tabla 1 es posible observar que $\mathrm{H} 1 \mathrm{a}$, $\mathrm{H} 1 \mathrm{~b}$ y $\mathrm{H} 1 \mathrm{c}$, son aceptadas, por ende, el grado de cultura organizacional orientada al cliente (COC), el grado de comprensión y conocimiento sobre los clientes y/o consumidores (CC) y el grado de orientación al valor del cliente que tengan los empleados (GOCE) se relacionarían positivamente con el grado de orientación al cliente (OC) que tenga la empresa en la industria del consumo masivo; obteniendo correlaciones positivas de 0,819 0,895 y 0,463 , respectivamente. Es posible observar correlaciones fuertes en el caso de COC y CC, lo que permite inferir que una empresa "orientada al cliente" mostrará mayores niveles de cultura organizacional focalizada en el cliente y de conocimiento de éstos; input clave para mejorar la gestión de la cartera de clientes desde una perspectiva de rentabilidad a largo plazo con el fin de potenciar la relación cliente-empresa.

La hipótesis $\mathrm{H} 1$ es aceptada, es decir, el grado de orientación al valor del cliente (OC) se relacionaría positivamente con el desempeño organizacional (DO) en una empresa del sector del consumo masivo. Presentando una correlación moderada de 0,583 , el grado de relación puede explicarse porque básicamente en la variable DO podrían estar influyendo otros factores, distintos a la OC, tanto internos como externos del negocio. 
Las Hipótesis $\mathrm{H} 2 \mathrm{a}, \mathrm{H} 2 \mathrm{~b}, \mathrm{H} 2 \mathrm{c}$ y $\mathrm{H} 2 \mathrm{~d}$ son corroboradas, debido a que se encontraron relaciones positivas entre el grado de cultura orientada al uso de TI (COATI) y el perfil de los empleados en relación al uso de TI (PEATI) tanto con la facilidad percibida de uso de TI (FUATI) como con la utilidad percibida de uso de TI (UPATI). Las correlaciones fueron de $0,297,0,637,0,741$ y 0,815 respectivamente. Cabe destacar la relación fuerte y positiva existente entre PEATI y UPATI, lo cual implicaría que empleados con una mayor orientación al uso de tecnologías percibirán mayor utilidad en el uso de éstas.

Las hipótesis $\mathrm{H} 2 \mathrm{e}$ y $\mathrm{H} 2 \mathrm{f}$ se verifican, encontrándose relaciones positivas entre el grado de utilidad percibida de uso de TI (UPATI) y facilidad percibida de uso (FUATI) con la actitud hacia el uso de TI (AUATI). Las correlaciones fueron de 0,788 y 0,496 respectivamente. Esto permite establecer que se comprueba lo expuesto por el Modelo TAM de Davis (1989).

La hipótesis $\mathrm{H} 2 \mathrm{~g}$ también es verificada, es decir, la actitud que se tenga hacia el uso de TI (AUATI) se relacionaría positivamente con el grado de adopción de TI. El coeficiente de correlación fue de 0,770 ; es decir, mientras más positiva sea la actitud que el empleado tiene en torno al uso de las tecnologías, mayor será el uso de las herramientas de tecnología.

La hipótesis $\mathrm{H} 2$ no se acepta, al mostrar una relación positiva no significativa entre el grado de adopción de tecnología de información ( $\mathrm{TI}$ ) con el grado de orientación al valor del cliente (OC), puesto que la correlación fue de 0,595 .
Finalmente, la hipótesis $\mathrm{H} 3$ es corroborada, es decir, el grado de adopción de tecnologías de información (TI) se relacionaría positivamente con el desempeño organizacional (DO) presentando una correlación moderada de 0,501 . Esto implicaría que a mayor grado de adopción de TI, el desempeño organizacional de la empresa mejoraría.

\subsection{Satisfacción del Cliente}

En la segunda fase de la investigación, la muestra consistió en: 1) 105 consumidores (14,3\% Hombres; $85,7 \%$ Mujeres) entre 18 y 24 años (70\%) con educación universitaria incompleta $(64,8 \%)$ para la "marca 1" la cual era de principal uso femenino $y, 2) 102$ consumidores $(100 \%$ hombres) entre 18 y 24 años $(76,5 \%)$ con educación universitaria incompleta $(75,6 \%)$ para la "marca 2" la cual era de uso exclusivo masculino.

El Alfa de Cronbach para el constructo Satisfacción del Cliente fue de 0,921 mostrando consistencia interna. El análisis factorial exploratorio arrojó que los 15 indicadores se agruparon en un solo factor mostrando unidimensionalidad de la escala.

Los resultados sobre Satisfacción del Cliente (SC), se indican en la Tabla 3 Se detallan indicadores y medias del constructo SC, siendo los promedios generales del constructo de 4.37 y 4.51 para la marca 1 y 2 respectivamente. Estos promedios permiten inferir que los clientes tienen niveles "bajos" de satisfacción tendientes a la "indiferencia" respecto de los productos que la empresa de consumo masivo en estudio comercializa. 
Orientación al Cliente, Tecnologías de Información y Desempeño...

Valenzuela Fernández, Leslier Maureen y Martínez Troncoso, Carolina Andrea

\section{Tabla 3 \\ Media por Indicador \\ Constructo Satisfacción del Cliente}

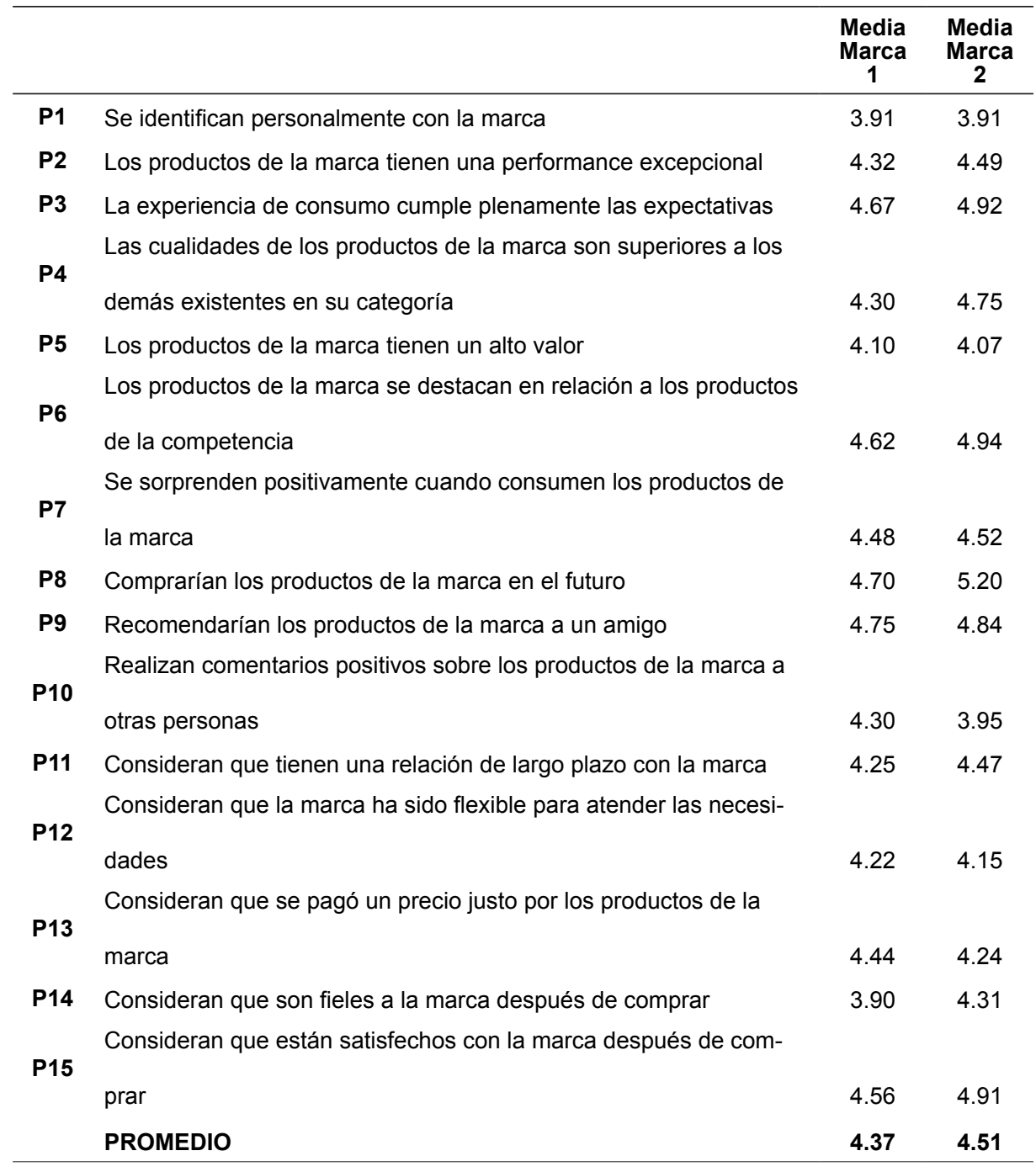

Fuente: Elaboración Propia 
En resumen, los resultados descritos anteriormente indican que la OC influye positiva y moderadamente en el DO, por lo que gestionar en torno al valor de los clientes puede marcar diferencias al permitir una distribución más eficiente de los recursos de marketing y ventas en los clientes más rentables. El horizonte de tiempo dentro de dicha estrategia es fundamental, ya que no sólo interesa retener a los clientes que son rentables en el presente, sino también aquellos que lo son en el futuro. Para esto es útil el valor de tiempo de vida del cliente, lo cual se refiere a la suma de los ingresos obtenidos de los clientes de la empresa durante el tiempo de vida de las transacciones después de la deducción del costo total de atraer, vender y dar servicio a los clientes, tomando en cuenta el valor del tiempo del dinero (Hwang et al, 2004).

Asimismo, los resultados permitieron observar que desde la perspectiva de la oferta, efectivamente una empresa exitosa (multinacional) percibe su gestión en función del valor del cliente y percibe un nivel relativamente alto de adopción de tecnologías de información, pero estos constructos no necesariamente constituyen una fuente para generar inteligencia de negocio, de lo contrario los niveles de "satisfacción de los clientes" (SC) no habrían sido "indiferentes".

\section{Conclusiones}

Hoy en día es importante que una empresa genere mayor conocimiento sobre sus clientes en función de cómo se les está entregando la propuesta de valor (producto y servicio), cómo perciben lo recibido, cuál es su percepción sobre la experiencia en la relación cliente-empresa, y cómo estas relaciones con los clientes generan valor para el negocio de la organización. ¿Son todos los clientes valiosos? La respuesta es probablemente no.

Conocer cuáles son realmente los clientes rentables o con mayor potencial de rentabilidad o con valor estratégico (reputación, valor de referencia) es relevante para la organización. Enfocar los recursos escasos y las estrategias de marketing y ventas en esta cuota de clientes para incrementar su grado de satisfacción, retenerlos, hacerlos crecer en la relación y fidelizarlos permitirá a la organización ser eficiente y aumentar la probabilidad de rentabilizar su negocio.

Por lo mismo, la cultura organizacional debe estar orientada a incrementar el valor para los clientes y mejorar los resultados del negocio. Es decir, que se le dé mayor importancia a la coordinación inter-funcional (mayor orientación al cliente interno) para lograr mayor eficiencia en la creación de valor.

La cultura organizacional en conjunto con el perfil de los empleados deben estar alineados a la adopción de Tecnologías de Información (TI) para favorecer la adopción y percepción de utilidad de las $\mathrm{TI}$ y el desarrollo de competencias tecnológicas, puesto que es un aspecto que mejora su rendimiento en el trabajo realizando un menor esfuerzo gracias a la facilidad de uso tecnologías.

Finalmente, respecto a los niveles de satisfacción de los consumidores para las dos marcas de esta empresa bajo estudio, rondan en el nivel de "indiferencia" lo que implicaría que los consumidores no estarían percibiendo mejoras en el producto y servicio recibido, respecto de una orientación al cliente por parte de la empresa. Esto se debe a las brechas existentes entre el valor de lo que los oferentes creen estar entregando 
y lo que los demandantes perciben estar recibiendo. Es decir, la diferencia entre el valor percibido y recibido. Esta brecha podría ser explicada por dos principales razones, primero la sobre confianza o falta de autocrítica por parte del personal de la empresa y segundo, la alta exigencia de los consumidores hacia las marcas en un ambiente cada vez más competitivo. Por tanto, la no existencia de conciencia por parte de la empresa de la posible brecha entre valor percibido y recibido puede llevar a niveles de indiferencia o insatisfacción por parte de los consumidores finales.

Todo lo anterior conlleva a establecer que, se debe contar con una cultura orientada al cliente propagada por toda la organización. De esta forma, los empleados desarrollan un perfil orientado a incrementar el valor del cliente, desde la perspectiva de la oferta o empresa y desde la percepción de la demanda (cliente y consumidor). El impacto de la gestión de orientación al cliente y la adopción de las TI sobre los resultados organizacionales presentan un desafío para evaluar en otras industrias y países para comparar la influencia entre sectores y países latinoamericanos con el fin de evaluar distintos factores internos, en específico el impacto de las distintas culturas.

\section{Referencias bibliográficas}

Anderson, Eugene, Fornell, Claes, y Lehmann, Donald (1994), "Customer Satisfaction, Market Share, and Profitability". Journal of. Marketing, Año 58, №3, pp. 53-66.

Araya, Sergio, Chaparro, Julián, Orero, Alejandro y Joglar, Hernán (2007), "Sistemas y Tecnologías de Información y su relación con el Desempeño de Instituciones
Universitarias en el ámbito de Gestión Institucional: un estudio desde la Teoría de Recursos y Capacidades". International Conference on Industrial Engineering \& Industrial Management, 5-7 de Septiembre de 2007 (pp. 1805-1816).

Armstrong, Gary y Kotler, Philip (2009), Marketing: An Introduction, Upper Saddle River, NJ: Pearson Education.

Atuahene-Gima, Kwaku; Slater, Stanley y Olson, Eric (2005), "The Contingent Value of Responsive and Proactive Market Orientations from New Product Program Performance. Journal of Product Innovation Management". Año 22, pp. 464-482.

Baker, William y Sinkula, James (2007), "Does Market Orientation Facilitate Balanced Innovation Programs? An Organizational Learning Perspective". Journal of Product Innovation Management. Año $24, \mathrm{~N}^{\circ} 4$, pp. $316-$ 334.

Baxter, Roger y Matear, Sheelagh (2004), "Measuring Intangible Value in Business-To-Business Buyer-Seller Relationships: An Intellectual Capital Perspective". Industrial Marketing Management. Año 33, pp. 491-500.

Berger, Paul D., Bolton, Ruth N., Bowman, Douglas, Briggs, Elten, V. Kumar, A. Parasuraman y Creed, Terry (2002), "Marketing Actions and the Value of Customer Assets: A Framework for Customer Asset Management". Journal of Service Research. Año 5, №1, pp. 39-55.

Brady, Michael y Cronin, Joseph (2001), "Customer Orientation: Effects on Customer Service Perceptions and Outcome Behaviours". Journal of Service Research, Año 3, N³, pp. 241-51.

Brady, Michael, Cronin, Joseph y Brand, Richard (2002), "Performance-only measurement of service quality: A replication and extension". Journal of Business Research. Año 55, N¹, pp. 17-31. 
Brown, Tom; Mowen, John; Donavan, Todd y Licata, Jane (2002), "The Customer Orientation of Service Workers: Personality Trait Effects on Self-and Supervisor Performance Ratings". Journal of Marketing Research. Año 39, pp. 110-119.

Brynjolfsson, Erik y Hitt, Lorin (1996), "Paradox Lost? Firm-Level Evidence on the Returns to Information Systems Spending". Management Science. Año 42, N, pp. 541-558.

Churchill, Gilbert; Ford, Neil; Hartley, Steven y Walker, Orville (1985), "The Determinants of Salesperson Performance: A Meta-Analysis". Journal of Marketing Research. Año 22, pp. 103-18.

Coley, Linda, Mentzer, John y Cooper, Martha (2010), "Is "Consumer Orientation" a Dimension of Market Orientation in Consumer Markets?". Journal of Marketing Theory and Practice. Año $18, N^{\circ} 2$, pp. 141-154.

Danneels, Erwin (2002), "The Dynamics of Product Innovation and Firm Competences“. Strategic Management Journal. Año $23, N^{\circ} 12$, pp. 1095-1121.

Davis, Fred (1989), "Perceived usefulness, perceived ease of use, and user acceptance of information technology". MIS Quarterly, Año 13, №3, pp. 319340.

Deeds, David y Rothaermel, Frank (2003), "Honeymoons and liabilities: the relationship between age and performance in research and development alliances". Journal of Product Innovation Management. Año 20, №6, pp. 468-85.

Deshpande, Rohit y Webster, Frederick (1993), "Organizational culture and marketing: Defining the research agenda". Journal of Marketing. Año 53, №1, 3-15

Elg, Ulf (2002), "Inter-Firm Market Orientation: Its Significance and Antecedents in Distribution Networks". Journal of Marketing Management. Año 18, Nㅜㄱ, pp. 633-656.
Flint, Daniel y Woodruff, Robert (2001), "The initiators of changes in customers desired value". Industrial Marketing Management. Año 30, pp. 321-337.

Fullan, Michael (1999), Change forces: The sequel. Philadelphia, PA: Falmer Press.

Frankec, George y Park, Jeong-Eun (2006), "Salesperson Adaptive Selling Behaviour and Customer Orientation: A Meta-Analysis". Journal of Marketing Research. Año 48, pp. 693-702.

Frishammar, Johan (2005), "Managing Information in New Product Development: A Literature Review". International Journal of Innovation and Technology Management. Año 2, N³, pp. 259-275.

García, Esteban; Rialp, Alex y Rialp, Joseph (2007), "Tecnologías de la Información y la Comunicación (TIC) y Crecimiento de la empresa". Nuevos Productos, Nuevos Mercados y Nuevas Formas de Internacionalización. Año $83, N^{\circ} 8$, pp. 125-145.

Gatignon, Hubert y Xuereb, Jean-Marc (1997), "Strategic Orientation of the Firm and New Product Performance". Journal of Marketing Research. Año 34, №2, pp. 77-90.

ljaz, Awais y Mehmood, Saqib (2011), "The Influence of Customer Orientation on Customer Relationship in Telecom Services". Information Management and Business Review. Año $3, \mathrm{~N}^{\circ} 4$, pp. 222-227.

Homburg, Christian; Müller, Michael y Klarmann, Martin (2011), "When Should the Customer Really Be King? On the Optimum Level of Salesperson Customer Orientation in Sales Encounters". Journal of Marketing. Año $75, \mathrm{~N}^{\circ} 55$, pp. 55-74

Hoque, Zahirul (2004), "A contingency model of the association between strategy, environmental uncertainty and performance measurement: Impact 
Orientación al Cliente, Tecnologías de Información y Desempeño... Valenzuela Fernández, Leslier Maureen y Martínez Troncoso, Carolina Andrea

on organizational performance". International Business Review. Año 13, pp. 485-502.

Hortinha, Paula; Lages, Carmen y Lages, Luis (2011), "The TradeOff between Customer and Technology Orientations: Impact on Innovation Capabilities and Export Performance". Journal of International Marketing. Año 19, $\mathrm{N}^{\circ} 3$, pp. 36-58

Hunter, Gary y Perreault, William (2007), "Making Sales Technology Effective". Journal of Marketing. Año 71, pp. 16-34.

Hwang, H., Jung, T., \& Suh, E. (2004), An LTV model and customer segmentation based on customer value: a case study on the wireless telecommunication industry. Expert systems with applications, 26(2), 181-188.

Jaramillo, Jorge y Grisaffe, Douglas (2009), "Does Customer Orientation Impact Objective Sales Performance? Insights from a Longitudinal Model in Direct Selling". Journal of Personal Selling \& Sales Management. Año 29, N², pp. 67-178.

Jiménez, Ana y Martínez, María (2006), "La influencia de las TIC en la distribución comercial: Implicaciones estratégicas para la gestión promocional minorista". Revista Iberoamericana de Ciencia, Tecnología, Sociedad e Innovación. $\mathrm{N}^{\circ} 7$.

Jones, Eli; Busch, Paul y Dacin, Peter (2003), "Firm Market Orientation and Salesperson Customer Orientation: Interpersonal and Intrapersonal Influences on Customer Services and Retention in Business-to-Business Buyer-Seller Relationships". Journal of Business Research. $N^{\circ} 56$, pp. 323-340.

Kaplan, Robert y Norton David (1996), "Using the balanced scorecard as a strategic management system". Harvard Business Review. Año 74, №1, pp. $75-85$,
Kirca, Ahmet; Jayachandran, Satish y Bearden, William (2005), "Market Orientation: A Meta-Analytic Review and Assessment of Its Antecedents and Performance". Journal of Marketing. Año 69, $\mathrm{N}^{\circ} 4$, pp. 24-41

Kohli, Rajiv y Devaraj, Sarv (2003), "Measuring information technology payoff: A meta-analysis of structural variables in firm level empirical research". Information Systems Research. Año 14, №2, pp. 127145.

Kotler, Phillip (1999), Marketing Management. Analysis, Planning, implementation and Control. Competition: Co-Creating Unique Value with New Jersey:Prentice Hall.

Kumar, Vineet.; Lemon, Katherine y Parasuraman, Arun. (2006), "Managing Customers for Value: An Overview and Research Agenda". Journal of Service Research. Año $9 \mathrm{~N}^{\circ} 2$, pp. 87-94.

Lam, Shun; Vandenbosch, Mark, Hulland, John y Pearce, Michael (2001), "Evaluating Promotions in Shopping Environments: Decomposing Sales Response into Attraction, Conversion and Spending Effects". Marketing Science. Año 20, №2, pp. 194-215.

Liang, Rong-Da, Tseng, Hsing-Chau y Lee, Yun-Chen (2010), "Impact of Service Orientation on Frontline Employee Service Performance and Consumer Response". International Journal of Marketing Studies. Año 2, $\mathrm{N}^{\circ} 20$, pp. 67-74

Macintosh, Gerrard (2007), "Customer Orientation, Relationship Quality, and Relational Benefits to the Firm". Journal of Services Marketing. Año 21, $N^{\circ} 3$, pp. 150-57.

Meléndez, Antonio y Moreno, Aurora (2012), Gestión de relaciones con clientes como iniciativa estratégica: implementación en hoteles. Revista Venezolana de Gerencia (RVG). Año 60, pp. 587-610.

Mukhopadhyay, Tridas; Kekre, Sunder y Kalathur, Suresh (1995), "Business 
value of information technology: A study of electronic data interchange". MIS Quarterly. Año 19, №2, pp.137156.

Narver, John y Slater, Stanley (1990), "The effect of a market orientation on business profitability". Journal of Marketing. Año 54, pp. 20-35

Oliver, Richard (1997), Satisfaction: a behavioural perspective on consumer. New York: McGraw-Hill.

Orantes, Sandra (2011), "Viabilidad del Modelo de Aceptación de la Tecnología en las empresas mexicanas: Una aproximación de las actitudes y percepciones de los usuarios de las tecnologías de la información". Revista Digital Universitaria. Año $12, N^{\circ} 1$.

Plank, Richard y Reid, David (1994), "The Mediating Role of Sales Behaviors: An alternative Perspective of Sales Performance and Effectiveness". Journal of Personal Selling \& Sales Management. Año 14, pp. 43-56.

Porter, Lyman y Lawler, Eduard (1968), Managerial Attitudes and Performance. Richard D. Irwin, Hommewood

Prahalad, Coimbatore Krishnarao y Ramaswamy, Venkat (2004), The Future of Customers. Boston: Harvard Business School Press.

Reinartz, Werner; Krafft Manfred, y Hoyer, Wayne (2004), "The customer relationship management process: its measurement and impact on performance". Journal of Marketing Research. Año 16, pp. 293-305.

Reinartz, Werner y Kumar, Vipin (2003), "The impact of customer relationship characteristic TIC's on profitable lifetime duration". Journal of Marketing. Año 67, pp. 77-99.

Riascos, Sandra, Ávila, Gloria y Quintero, Diana (2009), "Las TIC en el aula: percepciones de los profesores universitarios". Informática Educativa. Año 12, №3, pp. 133-157.
Shah, Denish; Rust, Roland; Parasuraman, A., Staelin, Richard y Day, George (2006), "The Path to Customer Centricity". Journal of Service Research. Año 9, pp. 113-124.

Slater, Staley y Olson, Eric (2001), "Marketing Contribution to the Implementation of Business Strategy: An Empirical Analysis". Strategic Management Journal. Año 22, pp. 1055-1067.

Valenzuela, Leslier y Castillo, Eduardo (2007), "El Cambio del Marketing en Dirección a una Nueva Era de Gestión Estratégica Centrada en el Cliente". Revista de Estudos de Administração. Año 15, pp. 97-108.

Valenzuela, Leslier (2007), "La gestión del valor de la cartera de clientes y su efecto en el valor global de la empresa: diseño de un modelo explicativo como una herramienta para la toma de decisiones estratégicas de marketing. (Tesis Doctoral)". Universidad Complutense de Madrid, Madrid, España.

Valenzuela, Leslier (2010), "Marketing orientado al cliente y ética empresarial: efectos sobre el valor de la cartera". Estudios de Administración. Año $17, \mathrm{~N}^{\circ} 2$, pp. $1-24$.

Valenzuela, Leslier; Mulki, Jay y Jaramillo, Jorge (2010), "Impact of Customer Orientation, Inducements and Ethics on Loyalty to the Firm: Customers Perspective". Journal of Business Ethics. Año 93, №2, pp. 277-291.

Valenzuela, Lesliery Martínez, Carolina(2012), "Las tecnologías de información y el desempeño organizacional. Propuesta de un modelo explicativo". Trend Management. Año 14, $\mathrm{N}^{\circ} 4$ pp. 60-66.

Valenzuela, Leslier y Torres, Eduardo (2008), "Gestión empresarial orientada al valor del cliente como fuente de ventaja competitiva". Propuesta de un modelo explicativo. Estudios Gerenciales. Año 24, No 109, pp. 65-86.

Yadav, Manjit y Varadarajan Rajan (2005), "Understanding Product Migration 
Orientación al Cliente, Tecnologías de Información y Desempeño...

Valenzuela Fernández, Leslier Maureen y Martínez Troncoso, Carolina Andrea

to the Electronic Marketplace: A Conceptual Framework". Journal of Retailing. Año 81. №2, pp. 125-40.

Yalcinkaya, Goksel; Calantone, Roger y Griffith, David (2007), "An Examination of Exploration and Exploitation Capabilities: Implications for Product Innovation and Market Performance". Journal of International Marketing. Año $15, \mathrm{~N}^{\circ} 4$, pp. 63-93.

Yang, Hui-O y Fu, Hsin-Wei (2007), "Creating and Sustaining Competitive Advantages of Hospitality Industry". Journal of American Academy of Business. Año 12, №1, pp. 113-119.

Zablah, Alex; Franke, George; Brown, Tom y Bartholomew, Darrell (2012), "How and When Does Customer Orientation Influence Frontline Employee Job Outcomes? A Meta-Analytic Evaluation". Journal of Marketing. Año 76, pp. 21-40

Zeithaml, Valarie (1988), "Consumer Perceptions of Price, Quality, and Value: A Means-End Model and Synthesis of Evidence". Journal of Marketing. Año 52, №3.

Zhou, Kevin Zheng; Yim, Chi Kin (Bennett) y Tse, David (2005), "The effects of strategic orientations on technologyand market-based breakthrough innovations". Journal of Marketing. Año $69, \mathrm{~N}^{\circ} 2$, pp. 42-60. 